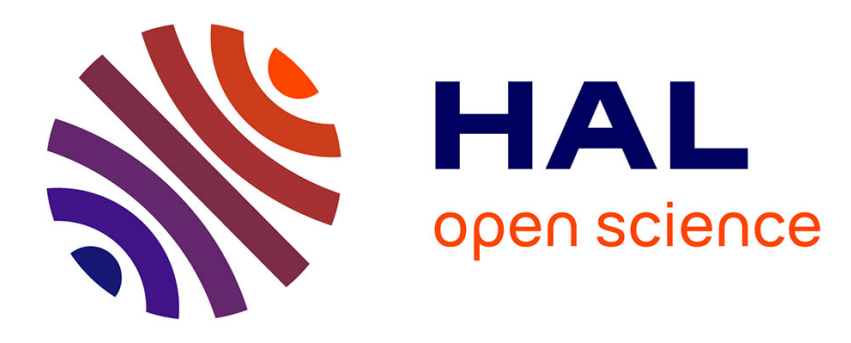

\title{
SQUEEZING IN BISTABLE OPTICAL SYSTEMS
}

\author{
S. Reynaud, E. Giacobino
}

\section{To cite this version:}

S. Reynaud, E. Giacobino. SQUEEZING IN BISTABLE OPTICAL SYSTEMS. Journal de Physique Colloques, 1988, 49 (C2), pp.C2-477-C2-482. 10.1051/jphyscol:19882112 . jpa-00227623

\section{HAL Id: jpa-00227623 https://hal.science/jpa-00227623}

Submitted on 1 Jan 1988

HAL is a multi-disciplinary open access archive for the deposit and dissemination of scientific research documents, whether they are published or not. The documents may come from teaching and research institutions in France or abroad, or from public or private research centers.
L'archive ouverte pluridisciplinaire HAL, est destinée au dépôt et à la diffusion de documents scientifiques de niveau recherche, publiés ou non, émanant des établissements d'enseignement et de recherche français ou étrangers, des laboratoires publics ou privés. 


\title{
SQUEEZING IN BISTABLE OPTICAL SYSTEMS
}

\author{
S. REYNAUD and E. GIACOBINO
}

Laboratoire de Spectroscopie Hertzienne de I'ENS, Université Pierre et Marie Curie, 24, Rue Lhomond, F-75231 Paris Cedex 05, France

Résumé : Il est bien connu que les systèmes optiques bistables peuvent fournir des etats "comprimés" du champ électromagnétique. On peut en principe atteindre une "compression" parfaite au voisinage du seuil de bistabilité. Nous montrons que l'on peut calculer les fluctuations quantiques du champ émis par un traitement "classique" dans lequel toutefois les fluctuations du vide sont correctement prises en compte. L'avantage de cette approche est sa simplicité conceptuelle qui permet de dégager une interprétation physique claire des spectres de fluctuations pour la "compression optimale" et pour la réduction du bruit d'intensité.

Abstract : It is known that optical bistable systems are capable of generating squeezed states of light. Perfect squeezing can even be achieved near the bistability threshold. We show that it is possible to obtain the quantum fluctuations of the outgoing field by a "classical" treatment provided that the vacuum fluctuations are properly accounted for. This approach allows us to give a simple physical interpretation to the Eluctuation spectra computed for optimum squeezing and for intensity squeezing.

With fluctuations equal to the minimum allowed by quantum mechanics, Glauber's coherent states / $1 /$ of the electromagnetic field have long been considered the least "noisy" light that one was able to produce. It is for example the case of the light emitted by a well stabilized laser in a noise frequency range far away enough from zero frequency. The quantum mechanical limit on the fluctuations of light stems from Heisenberg principle : the two quadrature components $a_{1}$ and $a_{2}$ of an electromagnetic field $a_{1} \cos \omega t+a_{2}$ sinwt are quantum mechanical non commuting operators and their uncertaint. $y$ product has a lower limit. For a coherent state, these uncertainties on $a_{1}$ and $a_{2}$ are equal, and equal to the allowed minimum. squeezed states of light are also minimum uncertainty states, but are characterized by different quantum fluctuations on the two quadrature components. In principle the fluctuations on one of them can be made arbitrarily small, and for more than a decade, a large amount of theoretical work has been devoted to the search and study of processes (usually non linear interactions) transforming coherent light into squeezed light.

Since 1985, several experiments have successfully prepared squeezed light $/ 2-6 /$. Among the proposed processes, optical bistability has been theoretically predicted to yield squeezing : absorptive or dispersive bistability in a two level system $/ 7-9 /$ was shown to give squeezing inside 
the cavity. However, after it was realized that much better squeezing could be obtained outside the cavity /10-12/, very good squeezing factors have been predicted near the bistability threshold /13-15/. Experiments/5/16/ have confirmed the existence of squeezing in the bistable regime.

In this paper, we want to discuss of the physical origin of squeezing in optical bistability. We will concentrate on the simplest model, a pure Kerr medium placed inside a single ended ring cavity.

\section{I - A QUALITATIVE PICTURE OE SQUEEZING IN OPTICAL BISTABLE SYSTEMS}

We consider a coherent field which enters an optical ring cavity containing a Kerr medium, whose index of refraction exhibits a linear dependence on the light intensity in the cavity. We shall use a phase space representation in which the coordinates are $a_{1}$ and $a_{1}$, the quadrature components of the field $a_{1}$ coswt $+a_{2}$ sinwt. Because of the quantum uncertainty on $a_{1}$ and $a_{2}$, a field is represented by some probability distribution in phase space. For a coherent field, the fluctuations on the two quadratures are equal, the probability distribution has the shape of a disk (Fig. 1, (a)).

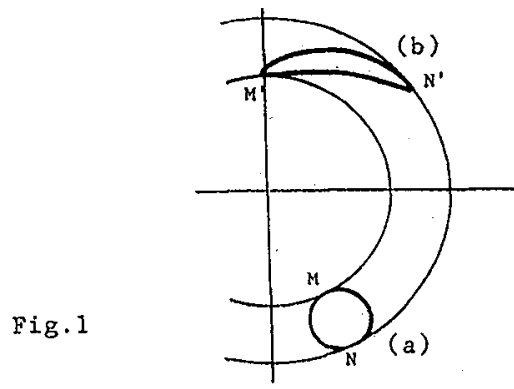

Because of the interaction with the kerr medium in the cavity, the field undergoes a phase rotation which depends on the intensity : all the parts of the disk are not rotated by the same amount and the probability distribution is distorted (Fig. 1,(b)) /18/. A few properties of the new probability distribution can be inferred very simply :

i) the two extreme points (in the direction of the field amplitude) of the initial probability distribution $M$ and $N$ move on two circles centered on the origin (Eig. 1 ), giving $M^{\prime}$ and $N^{\prime}$. Since the rotation angles are different, the distance $\mathrm{M}^{1} \mathrm{~N}^{\prime}$ is greater than $\mathrm{MN}$. But the probability distribution remains confined between the two circles, which means that the intensity distribution is not modified.

ii) this geometrical transformation obviously conserves the area. Now, as one of the dimensions of the new probability distribution is increased (M'N'), another one has to be decreased. In other words, squeezing is expected on one particular quadrature component, corresponding to the smallest diameter of the probability distribution.

iii) when one gets close to the bistability threshold, it is well known that a critical divergence of the fluctuations occurs : the larger dimension of the distribution goes to infinity, and so the smaller one goes to zero. 
In the next section, we present the calculations of the quantum fluctuations. We will see that they confirm our simple predictions for the noise frequency at $\omega=0$. In particular, since the photon number is conserved when measured over long times, no intensity squeezing can occur at $\omega=0$. But we will see also that the intensity noise may be squeezed at some non zero frequencies. This effect is associated with a temporal redistribution of the incident photons on a time scale of the order of the cavity storage time.

\section{II - EQUATIONS FOR THE FIELD ELUCTUATIONS}

In the good cavity limit, the relationship between the incident field $F^{\prime n}$ and the intracavity field $E$ (Fig. $2 a$ ) can be written as :

$$
E(\gamma+\iota p)=t F^{1 n}
$$

where $\varphi$ is the round trip phase accumulated by the field in the cavity (including the effect of the Kerr medium) and $r$ the cavity width connected to the reflexion and transmission amplitude coefficients $r$ and $t$ of the coupling mirror by :

$$
r=1-r ; t=\sqrt{2 \gamma}
$$

Denoting :

$$
X=|E|^{2} \quad ; \quad Y=\left|E^{1 n}\right|^{2}
$$

the intracavity and incident intensities, one gets the classical solution :

$$
2 \gamma Y=X\left(\gamma^{2}+\varphi^{2}\right)
$$

which yields the usual bistability curve if $\varphi$ exhibits a linear dependence on $\mathrm{X}$ (Fig. $2, \mathrm{~b}$ corresponds to $\varphi_{0}=-5 \gamma$ ) :

$$
\varphi=p_{0}+f X
$$

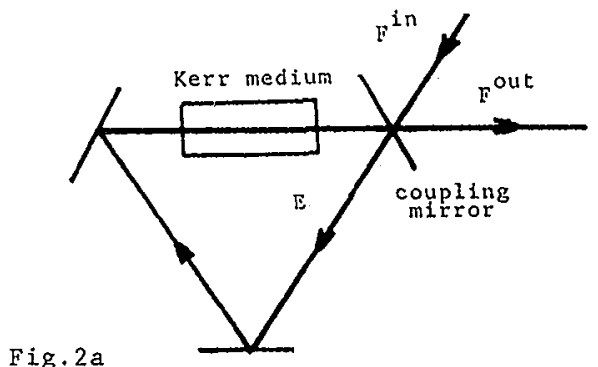

Fig. 2b

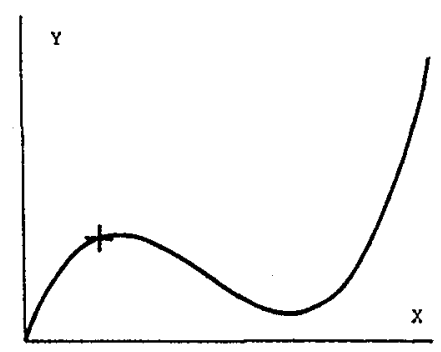

In the following, we will characterize a working point on the bistability curve by the slope parameter $p=\frac{d Y}{d X}$. Derivating (4) with respect to $X$ 
gives a relationship between $\mathrm{p}$ and $\mathrm{x}$ :

$$
2 \gamma p=\gamma^{2}+\varphi^{2}+2 \varphi f X
$$

The vicinity of a bistability threshold wiil be associated to a positive value of $p$ close to zero (negative values are associated to the unstable branch).

The field fluctuations can be computed very simply using the following "semi-classical" approach $/ 19 /$. The dynamics of small fluctuations are described by linearizing the classical equations of motion in the vicinity of the stationnary working point (same procedure as in linear stability analysis). Then we consider that these field fluctuations are driven by the fluctuations of the incident field which enter the cavity through the coupling mirror.

Taking the Fourier transforms of the field fluctuations, one gets in this manner the following Iinear relation:

with

$$
t\left[\begin{array}{c}
\delta F^{1 n} \\
\delta F^{1 n *}
\end{array}\right]=M\left[\begin{array}{l}
\delta E \\
\delta E^{*}
\end{array}\right]
$$

$$
M=\left(\begin{array}{ccc}
-\iota \omega \tau+\gamma+\iota \varphi+\imath \mathrm{fX} & \imath f X \\
-\imath \mathrm{fX} & -\imath \omega \tau+\gamma-\imath \varphi-\imath \mathrm{EX}
\end{array}\right)
$$

where $\omega$ is the noise frequency and $\tau$ the round trip time (the mean fiela $\bar{E}$ has been chosen to be real).

We now want to get the fluctuations of the output field $F^{\text {out }}$. In order to get this field, one can simply write the usual transmission-reflexion equation on the coupling mirror assuming a high reflexion coefficient :

$$
F^{0 u t}=t E-F^{j n}
$$

Then the fluctuations of the output field are obtained by eliminating the intracavity field :

$$
\begin{aligned}
& \delta F^{0 u t}=t \delta E-t \delta F^{1 n} \\
& {\left[\begin{array}{ll}
\delta & F^{0 u t} \\
\delta & F^{0 u t}
\end{array}\right]=\left(\begin{array}{llll}
2 & \gamma M^{-1}-1
\end{array}\right)\left[\begin{array}{ll}
\delta & F^{1 n} \\
\delta & F^{1 n *}
\end{array}\right] }
\end{aligned}
$$

The transfer matrix $\left(2 \mathrm{rM}^{-1}-1\right)$ contains all the information necessary to get the fluctuation spectra. For example, the optimum squeezing, that is to say the smallest diameter of the probability distribution, can be obtained as :

$$
S_{o p t}(\omega)=|\lambda|^{2}
$$

where $\lambda$ is the smaller eigenvalue of the transfer matrix. The intensity squeezing can be computed by writing the fluctuations of the output intensity : 


$$
\delta I^{0 u t}=\bar{F}^{0 u t} \delta F^{0 u t}+\bar{F}^{0 u t *} \delta F^{0 u t}
$$

We now discuss the fluctuation spectra obtained in this manner.

\section{III - ELUCTUATION SPECTRA}

Figure 3 shows the spectra corresponding to the optimum squeezing (curve a) and to the intensity squeezing (curve b) at a working point close to the bistability threshold on the lower branch $(\mathrm{p} / \gamma=0.3$ corresponding to the cross in (Fig. 2.. b) . It clearly appears that a good squeezing is obtained on a frequency range of the order of the cavity bandwidth. The best squeezing corresponds to the null frequency.

Fig. 3

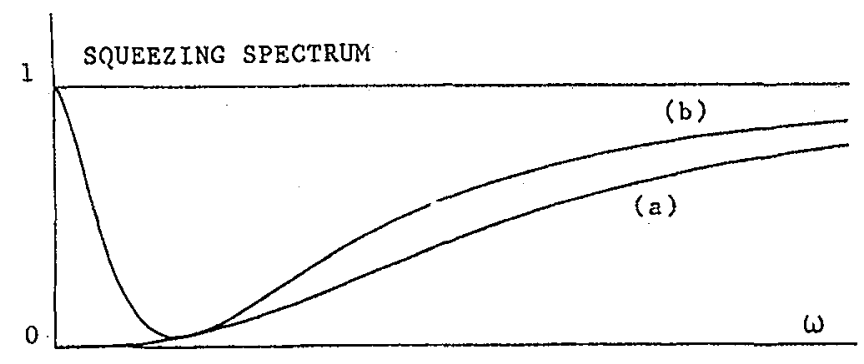

As expected from the qualitative discussion, there is no intensity squeezing at $\omega=0$ (curve $b$ starts from the value 1). However, an appreciable intensity squeezing is obtained at non zero frequencies. This can be interpreted as resulting from a rotation of the probability distribution when the analysis frequency is varied. For some particular frequency, the curve b gets tangent to the curve $a$, which means that the squeezed quadrature component is just the field amplitude at this frequency.

IV - EEFECT OE EXTRA LOSSES
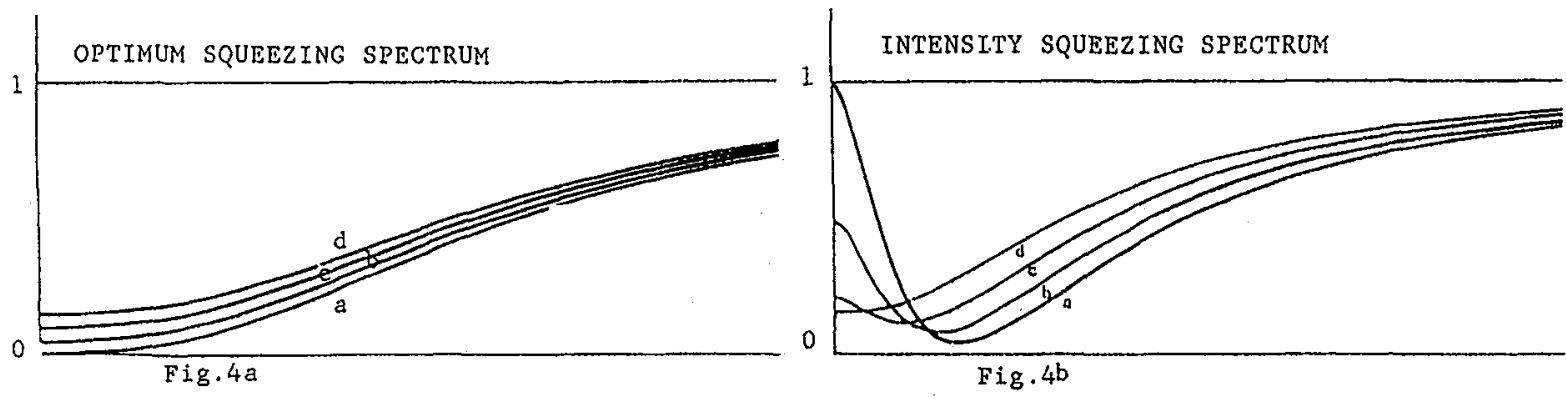

Up to now, we have considered that the only source of noise for the intracavity field is the scape through the coupling mirror. In order to discuss the effect of extra losses, we will use the simple model of loss through a second mirror (loss coefficient $r^{\prime}$ ). 
Figure $4 a$ shows some spectra obtained in this manner for the optimum squeezing with increasing values of the extra losses $\left(\gamma /\left(\gamma+\gamma^{\prime}\right)=0,5 \%\right.$, $10 \%, 15 \%$ ). As it is always the case, the presence of extra losses tends to decrease the amount of squeezing $/ 17 /$.

Figure $4 \mathrm{~b}$ shows the spectra obtained in the same conditions for the intensity squeezing. It appears that extra losses allow a better intensity squeezing at zero frequency. This rather paradoxical result can be understood by considering that a non zero value of $\gamma^{\prime}$ induces a rotation of the mean field $F^{\circ}{ }^{t}$. This rotation can put the squeezed quadrature component along the direction of the field amplitude and is therefore the cause of the unexpected intensity squeezing at zero frequency.

\section{CONCLUSION}

We have reviewed in what conditions a very simple optical bistable system can produce squeezed states of light. The novel point we wish to emphasize is that it allows to design passive devices "eating" the intensity noise of an incident laser.

\section{REFERENCES}

/1/ Glauber R.J., Phys. Rev. 131 (1963) 2766.

/2/ Slusher R.E., Hollberg L.W., Yurke B., Mertz J.C. and Valley J.F., Phys. Rev. Lett. 55 (1985) 2409.

/3/ Wu L.A., Kimble H.J., Hall J.L. and Wu H., Phys. Rev. Lett. 57 (1986) 2520 .

/4/ Shelby R.M., Levenson M.D., Perlmutter S.H., de Voe R.G. and Walls D.F., Phys. Rev. Lett. 57 (1986) 691.

/5/ Raizen M.G., Orozco L.A., Xiao M., Boyd T.L. and Kimble H.J. Phys. Rev. Lett 59 (1987) 198.

/6/ Heidmann A., Horowicz R.J., Reynaud S., Giacobino E., Fabre C. and Camy G., Phys. Rev. Lett. 59 (1987) 2555.

17/ Lugiato L.A. and Strini G., Opt. Commun. 41 (1982) 67.

$/ 8 /$ Lugiato I.A. and Strini G., Opt. Commun. 41 (1982) 447.

19/ Walls D.F. and Milburn G.J. in "Quantum Optics, Gravitation and Measurement Theory, ed. Meystre P. and Scully M.O., (Plenum New-York $1982)$.

/10/ Yurke B. Phys. Rev. A 29 (1984) 408.

$/ 11 /$ Collett J. and Gardiner C.W., Phys. Rev. A .30 (1984) 1386.

$112 /$ Gardiner C.W. and Savage C.M. Opt. Commun. 50 (1984) 173 .

/13/ Reid M.D. and Wails D.F., Phys. Rev. A 32 (1985) 396.

/14/ Coliett M.J. and Walls D.F., Phys. Rev. A 32 (1985) 2887.

115/ Min Xiao, Kimble H.J. and Carmichael H.J., J. Opt. Soc. Am. B 4 (1987) 1546 .

/16/ Orozco L.A., Raizen M.C., Min Xiao, Brecha R.J. and Kimble H.J., J. Opt. Soc. Am. 4 (1987) 1490 .

/17/ Reid M.D. and Walls D.F., Phys. Rev. A 31 (1985) 1622.

/18/ Kitagawa M. and Yamamoto Y., Phys. Rev. A 34 (1986) 3974.

119/ Reynaud. S., Fabre C. and Giacobino E., J. Opt. Soc. Am. B 4 (1987) 1520 . 